\title{
ВВЕДЕНИЕ И ПРИМЕНЕНИЕ ДИНАМИЧЕСКОЙ СОСТАВЛЯЮЩЕЙ ДАТЧИКОВ В СПЕЦИАЛИЗИРОВАННОЙ КОМПЬЮТЕРНОЙ СИСТЕМЕ
}

The introduction and application of the dynamic component of the sensor in a specialized computer system

\author{
Ухина А.B. ${ }^{1}$, Ситников T.B. ${ }^{1}$, Ситников B.C. ${ }^{2}$ (Ukhin A.V. Sitnikov T.V., Sitnikov V.S.) \\ 1,2 Одесский национальный политехнический университет, г. Одесса \\ 2 (ORCID iD is 0000-0003-3229-5096) \\ 2 E-mail: sitnvs@mail.ru
}

Copyright (C) 2014 by author and the journal "Automation technological and business - processes". This work is licensed under the Creative Commons Attribution International License (CC BY). http://creativecommons.org/licenses/by/4.0/

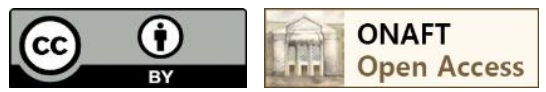

\section{DOI: $10.15673 /$}

\begin{abstract}
Аннотация
Рассмотрено введение и применение динамической составляющей датчиков в специализированной компьютерной системе, а так же ее расширение. Показано назначение и возможности таких датчиков. Предложена модель их применения в охранных, пожарных и в аварийных системах.
\end{abstract}

\begin{abstract}
Introduction and application of a dynamic component of sensors in specialized computer system, and also its expansion is considered. Appointment and possibilities of such sensors is shown. The model of their application in security, fire and in emergency systems is offered.
\end{abstract}

Ключевые слова

специализированная компьютерная система, «умный дом», статические датчики, динамические датчики, средства доставки, охранные системы, пожарные системы, аварийные системы.

В настоящее время внедрение специализированных компьютерных систем в различные сферы деятельности человека идет интенсивным образом. Даже современный дом невозможно представить без различных устройств, которые упрощают быт и делают проживание в нем комфортным, экономичным и безопасным. Наиболее прогрессивной концепцией взаимодействия человека (пользователя), например, с жилым пространством является специализированная компьютерная система «Умный дом». Эта специализированная система включает в себя измерения, анализ состояния и управления несколькими или всеми бытовыми устройствами распределено или централизованно, а также сбора информации о состоянии дома, с помощью системы датчиков [1-3].

В данной специализированной компьютерной системе обеспечивается ряд важных функций для жилого помещения, которые необходимы для нормального проживания (пребывания). Примерами этих функций служат: микроклимат, освещение, безопасность, своевременное реагирование на аварийные ситуации и т.д. Также решается вопрос согласованной работы различных по своему назначению устройств. Например, кондиционер не будет работать при включенном обогревателе, или во время проветривания комнаты. Это обеспечивает экономию ресурсов и освобождает человека от необходимости следить за всеми механизмами самостоятельно. Отпадает 
необходимость в нескольких пультах управления. Им на замену приходит универсальный пульт управления [2-3]. Жилец будет ознакомлен с состоянием в доме или с возникновением аварийной ситуации, даже если физически будет находиться далеко от него. Система сама оповестит его о непредвиденной ситуации.

Выше описанная система с успехом применяется и на современном производстве в полном объеме или частично.

Следует отметить, что построение подобных систем основано на использовании статической сети датчиков расположенных в разных местах дома и измеряющие или фиксирующие различные физические величины.

Однако, кроме констатации факта, например, срабатывания охранной системы или срабатывания датчиков воспламенения или затопления необходимо доуточнить уровень ущерба и показать состояние объекта аварии. Для таких целей предлагается ввести понятие динамических датчиков, которые могут появляться в местах срабатывания статистических датчиков.

Например, при срабатывании датчика разбития стекла или при срабатывании охранной подсистемы и проникновения в помещения целесообразно динамически поместить дополнительный датчик, например, видеокамеру, для передачи изображения владельцу. Разместить стационарно видеокамеру в каждом помещении дорого, да и не всегда необходимо. Та же ситуация возможно и при прорыве трубопровода и при возникновении пожара.

Динамическое размещение дополнительных датчиков возможно за счет роботехнических платформ квадрокоптеров различного вида или роботизированных тележек, которые на своем борту имеют дополнительные датчики [4-5]. Такой вариант размещение различных датчиков позволяет оперативно их перемещать по площади дома и производить дополнительные измерения или снимать дополнительную информацию, а в перспективе и отдавать команды исполнительным механизмам.

На сегодняшний день известны интеллектуальные датчики и “облако датчиков” [6-7]. Расширяя идею динамической составляющей датчиков специализированной компьютерной системы на другие объекты, можно привести варианты их применения.

Основное назначение динамической составляющей датчиков специализированной компьютерной системы получение дополнительной информации об интересующем объекте или явлении, измерения различных физических данных за счет перемещения в пространстве.

Примером объекта, где необходимы динамические датчики может служить сооружение, которое занимает большую территорию, которое необходимо тщательно охранять. Обычно для таких целей используется большой штат охраны, который довольно дорого содержать.

Структура объекта предусматривает статические датчики, которые расположены по периметру и внутри некоторых зон охраны для сигнализации о проникновении и сигнализации о пожаре. Статические датчики это первичный контур сигнализации.

Вторичный контур сигнализации может представлять собой мультикоптер или набор динамических датчиков, которые выстреливаются в случае принятия сигнала. В случае мультикоптера, он может вылететь в нужный квадрат, передать изображение на командный пункт и в случае необходимости сбросить набор динамических датчиков, определить направление распространения пожара или другой аварийной ситуации.

Динамический датчик в этом случае может представлять собой краткосрочный элемент питания, например, датчик температуры или движения, систему определения координат и микропроцессорную систему обработки и передачи информации по радиоканалу на сервер в зоне видимости, обычно не более 1-3 км. Спектр применения датчиков подобного типа очень широк. Ограничение - лишь стоимость и невозможность сбора информации при каких-либо условиях. Однако сегодняшние интегральные технологии позволяют упростить и удешевить эту проблему.

Применение динамических датчиков при тушении лесных пожаров очень своевременна. Если динамические датчики разбросать на некоторой территории, то можно собирать данные о температуре и влажности на контролируемой территории [8]. С помощью мультикоптера или другого летательного аппарата можно принимать информацию от набора датчиков, а при возникновении пожара еще и направление, и скорость, области активности пожара или наводнения. Дополнительные датчики могут измерять и степень загрязненности воды и воздуха, зараженности территории, радиационной обстановки и т.д.

Для охраны военных объектов могут использоваться датчики движения или комплекс датчиков, которые с большой скоростью смогут дать информацию о характере проникновения, примерной численности и характере противника. Анализируя спектр сигнала можно достаточно точно сказать о характере проникающей техники по датчикам движения и датчикам звука.

Динамические датчики найдут свое место и при ликвидации аварий и техногенных катастроф для оценки ситуации и измерения, физических полей при нежелательном присутствии человека. 
Ключевым недостатком такой системы является стоимость динамических датчиков. Обычно это одноразовые элементы, которые имеют ограниченный срок службы и их цена зависит от количества в изготавливаемой партии. Однако существуют области, в которых стоимость датчиков может быть оправдана.

Таким образом, предлагается новое видение конфигурации специализированной компьютерной системы при наличии динамической составляющей ее датчиком, которые могут перемещаться по контролируемому пространству для получения дополнительной информации, которая позволяет в реальном масштабе времени более адекватно и оперативно принять решение по ликвидации последствий непредвиденных обстоятельств. При этом доставка таких датчиков может быть осуществлена различным способом. Такая классификация датчиков позволяет на этапе проектировании строить заведомо оптимизированные и информативные системы, работающие в реальном масштабе времени, т.к. они вписываются в стандартные конфигурации специализированных компьютерных систем.

\section{Литература}

[1] R.C. Elsenpeter and T.O. Velte Build your own smart home. McGraw-Hill Companies, 2003;

[2] W. Harke Smart home.C.F. Muller Verlag, Heidelberg, 2004;

[3] S. Figaro, F. Leperou, M. Andreoni, B. Cauvy. Microsensors. Available: http://bde.polytechnice.fr/jahia/webdav/site/bde/shared/Utile/Cours/ELEC/ELEC4/projet_electronique_microsens ors_2008.pdf;

[4] IEEE Approves New IEEE 802.1aq Shortest Path Bridging Standard. Available: http://www.techpowerup.com/165594/ieee-approves-new-ieee-802-1aq-shortest-path-bridging-standard.html;

[5] For the future hydrogen economy, a tiny, self-powered sensor. Available: http://news.ufl.edu/archive/2006/05/forthe-future-hydrogen-economy-a-tiny-self-powered-sensor.html;

[6] Дж. Фрайден. Современные датчики. Справочник. Техносфера, 2005;

[7] К. Хадлстон. Проектирование интеллектуальных датчиков с помощью Microchip dsPIC. MК-Пресс, 2008;

[8] Overview of sensors and needs for environmental monitoring. Available: http://www.mdpi.net/sensors/papers/s5010004.pdf.

\section{References}

[1] R.C. Elsenpeter and T.O. Velte Build your own smart home. McGraw-Hill Companies, 2003;

[2] W. Harke Smart home.C.F. Muller Verlag, Heidelberg, 2004;

[3] S. Figaro, F. Leperou, M. Andreoni, B. Cauvy. Microsensors. Available: http://bde.polytechnice.fr/jahia/webdav/site/bde/shared/Utile/Cours/ELEC/ELEC4/projet_electronique_microsens ors 2008.pdf;

[4] IEEE Approves New IEEE 802.1aq Shortest Path Bridging Standard. Available: http://www.techpowerup.com/165594/ieee-approves-new-ieee-802-1aq-shortest-path-bridging-standard.html;

[5] For the future hydrogen economy, a tiny, self-powered sensor. Available: http://news.ufl.edu/archive/2006/05/forthe-future-hydrogen-economy-a-tiny-self-powered-sensor.html;

[6] D. Friden. Handbook of modern sensors. Technosphera, 2005;

[7] K. Hadkston. Designing intelligent sensors using Microchip dsPIC. MK-Press, 2008;

[8] Overview of sensors and needs for environmental monitoring. Available: http://www.mdpi.net/sensors/papers/s5010004.pdf. 